Aargau 80, 236, 239

Abgeordnetenhaus 53, 296-298, 301-303, 305, 306, 308-310, 312-314, 319-323

Abrogatives Referendum 43, 49, 51 Absinthverbot 222

Abstimmungen 11, 13, 14, 16, 18-21, 23-25, 27, 29, 47, 51, 59, 67, 68, 73-77, 79-81, 83, 90, 98, 110-112, $115,122,131-133,135,137,139$, $141,145,149,150,156-158,166$, $172,173,175,177,194,196,214$, 223, 235, 239, 259-261, 263, 265, $295,325,328$

Abstimmungsbeteiligung 49, 52, 173, 194, 218, 312, 325-327

Abstimmungskampf 52, 58, 82, 171, 179, 194, 197, 220, 328

Abstimmungsmehrheit 113, 127

Abstimmungstermine 217, Siehe Koppelung

Abstimmungsverhalten 52, 101, 261, 325, 328

Abwahl des Bürgermeisters 250

Abwahlen (Recall) 165

Abzockerei 233

Adenauer, Konrad 85

Advisory Referendum 169

Affirmative Action 75, 182, 183, 195, 196

Alaska 166, 173, 181, 189, 193, 194, 198, 200, 202

Allgemeine Volksinitiative 206, 207, 212

Alpeninitiative 220, 221

Alternative Liste 237

Ämterpatronage 33
Amtliche Obstruktion direktdemokratischer Initiativen 328

Amtseintragung 110, 170, Siehe Freie Stimmsammlung, Unterschriftensammlung

Amtszeitbeschränkungen der Parlamentsmitglieder 179

Annexion Österreichs 136, 140

Appenzell Ausserrhoden 74, 79, 80, 210, 217, 236

Appenzell Innerrhoden 74, 208, 217, 223

Arbeitsverbot illegaler Einwanderer 178

Arizona 83, 166, 174, 175, 177-179, $181,183,187,195,196,198,200$, 202

Arkansas 183, 189, 200, 202

Armeewaffen 232

Art. 146 GG 19, 20, 122

Atomenergie 239

Ausschaffungsinitiative 67, 220, 233

Ausschlusskatalog 25, 94, 103, 332, Siehe Haushaltsvorbehalt

Austritt aus dem Völkerbund 136, 139

Autobahnbau 177, 198

B-VG 241-257

Baden-Württemberg 298, 300, 307, 332

Bandendelikte 189

Bankgeheimnis 231

Basel-Landschaft 217, 232, 236

Basel-Stadt 217, 237, 238

Bauleitplanung 95, 332

Bauspar-Initiative 232

Bayerische Verfassung 25, 30 
Bayerischer Verfassungsgerichtshof 33, 128, 317, 329

Bayern 15, 16, 19, 24, 36, 40, 43, 44, 46, 50, 65, 95, 171, 218, 298, 307, $314,315,317$

Berlin 41-43, 48-50, 53, 57, 58, 60, 64, 94, 95, 138, 143, 149-151, 153, 162-164, 197, 295, 297-301, 303, 304, 306-315, 319, 321-323, 333

Berliner Doppelhürde 41, 53

Berliner Verfassungsgerichtshof 25, 310, 311

Bern 80, 210, 211, 237-240

Bestimmtheitsanforderungen an die Fragestellung im Bürgerentscheid 329

Beteiligung an Bürger- und Volksentscheiden 28

Beteiligungsquorum 22, 31, 40-45, 48-54, 56-60, 63, 64, 164, 264, 312

Beust, Ole von 96, 99

Bevölkerungsentscheide 19

Biometrische Pässe 229

Blocher, Christoph 66, 68, 224

Bond Referendum 168

Boykott 22, 30, 43-58, 64, 65, 156

Brandenburg 162, 298-302, 307, 311, 318, 323, 333

Braunschweig 46, 48

Bremen 16, 41, 47, 50, 51, 104, 105, 112, 298-300, 307, 315

Bremischer Staatsgerichtshof 112

Briefeintragung 110, 127

Budgetrecht 25, 26, 124, Siehe Haushalt

Bulgarien 228

Bundesgericht 67, 74, 80, 85-87, 186, 221, 222, 224, 225, 227, 237

Bundeskanzlei 213, 215
Bundespräsident 14, 241, 243, 245, 246, 252

Bundesrat 17, 71, 206, 216, 217, 221, 225, 228, 230-233, 246, 255

Bundesratsinitiativen 103

Bundesregierung 18, 243, 245, 254, 255

Bundestag 17, 30, 42, 61, 62, 85, 100, 110-114, 120, 245

Bundesverfassung 12, 67, 71, 74, 75, 79, 82, 87, 118, 205-209, 217-220, 222, 226, 229, 231, 235, 238, 241, 243, 246, 248-254, 282

Bundesverfassungsgericht 23, 25, 31 , $37,60,62,70,72,84,85,121,122$, 283

Bundesversammlung 67, 206, 207, 212-214, 216, 217, 226-230, 233-235

Bundeswehr 18

Bündnis 90/Die Grünen 35, 42, 65, 340

Bürgerantrag 299

Bürgerbegehren 14, 30, 49, 50, 94, 122 , 163, 173, 280, 303, 304, 318, 329-332

Bürgerentscheid 14, 17, 26, 30, 40, 41, 43, 48-50, 57, 60, 64, 94, 163, 164, 173, 296, 304, 329, 330, 332

Bürgerschaft 33, 50, 90-93, 95-108, 110-120, 129, 130, 161, 162, 219

Bürgerversicherung 27

Buß- und Bettag 50, 57

BVP 46

Carpool Lane 177

CDU 30, 35, 61, 90, 94-96, 98, 99, 101, 130

Centovalli 239

Chemnitz 151, 154, 155

Christliche Gemeinschaftsschule 36 
clausula rebus sic stantibus 116

clear and present danger 117

Colorado 83, 166, 174, 175, 177, 178, 180, 182, 183, 187, 190, 192, 196, 198, 200, 202

Comité Balladur 261

Community Colleges 192

Compulsory Referendum 168

Connecticut 184, 192, 200

Conseil constitutionnel 259, 262, 264-269

Constitutional Referendum 167

Convention Question 169

Constitutional Initiative 167

CSU 24, 30, 35, 36

de Gaulle, Charles 260, 261

Deckungsvorschlag 96, 125, 308, 319

Delaware 167, 200

Demagogie 23, 29, 91

Democratic Party 179

Demokratie als riskante Ordnung 31

Demokratie und Rechtsstaat 69

Demokratiedefizit der EU 272, 283

Demokratische Legitimation 16

Demokratische Partei 180, 185, 188

Demokratische Verfassungstradition 71

Deputationen 91

Die Linke 35

Diktatur 131-133, 135-138, 142, 157, 158

Direkte Demokratie 11-13, 17, 24-26, $28,29,34,35,40,65,68,69,74,75$, $81,90,92,99,122,165,205,218$, 243, 248, 251, 256, 257, 261, 271, 274, 284, 301, 305, 315, 316, Siehe Sachunmittelbare Demokratie

Diskontinuität 255
Diskriminierung 70, 74, 77, 85, 86, 224, 225

Distanzverlust 32

DNVP 154, 155

Dogma von der Gesetzessouveränität 266

Doppelmehr-Abstimmungen 223

Dreistufiges Modell der Volksgesetzgebung 93, 104

Dresden 61, 151

Dysfunktionalität von Quoren 54

Eheschließung 76, 183-186, 194, 195, 198, Siehe Homosexuelle

Einbindung des Parlaments 168, 171, 268, Siehe Parlament, Gegenvorschlag des Parlaments

Einbürgerungen $67,68,70,79,80,85$, 86, 219, 223-225, 238

Eine Schule für Alle 161

Einheit der Form oder Materie 216

Einheit der Verfassung 109

Einheitsinitiative 212

Einkommensteuer 175, 176, 203

Emmen 69, 70, 224

Enteignungsfragen 182

Entscheidung in eigener Sache 32, 33, 86

Erfolgswert 29, 61, 62, 65, 223

Ergänzungsfunktion $18,35,55,123$, 250, 271, 283, 301, 305

Erneuerbare Energien 193, 195

Erweitertes Referendum 209

Erweiterung der Personenfreizügigkeit 228

Erweiterungen der EU 263

Europäische Bürgerinitiative 280, 283

Europäische Menschenrechtskonvention $66,67,84,87,219,221,222$, 227,268 
Europäische Union 72, 271-275, 283

Europäischer Gerichtshof für Menschenrechte 67, 86

Europäischer Haftbefehl 37

Europäisches Bürgerbegehren 284

Europäisches Parlament 97, 111, 114, 274-276, 278, 279, 283

Ewigkeitsklausel 11, 12, 17, 20, 21, 72, $121,124,196,220,314$

Exekutive 17, 25, 124, 240, 261

Fakultatives Referendum 15, 173

Fakultatives Referendum 71, 168

FDP 30, 35, 96, 227

Finanzreferendum 207, 210

Finanzwirksame Abstimmungen 25, $26,32,33,104$

Florida 166, 173, 184, 196, 198, 200, 202

Föderatives Referendum 208

Forschung an embryonalen Stammzellen 191

Frankreich 32, 70, 103, 259, 260, 262, 264, 266, 268-270, 278

Französische Verfassung 259

Frauenstimmrecht 74

Freie Stimmsammlung 98, 110, 127

Führerkult 144

Funktionsvorbehalt 18, 19

Fürstenenteignung 22, 30, 43, 44, 56

GAL 94, 95, 99, 101, 103, Siehe Bündnis 90/Die Grünen

Gegenstände der politischen Willensbildung 301

Gegenvorschlag des Parlaments 49, $102,210,216,222,223,225,226$, $228,230,233,234,236$

Geheimheit der Abstimmung 29

Geheimheit der Wahl 146, Siehe Wahlgeheimnis
Geldeinfluss 171, 196, 199, 204

Geldgeber 181, 192, 198

Gemeinden 79-81, 86, 122, 134-136, $150,152-154,156,158,161,190$, $208,217,218,224,234,238-241$, $244,248,250$

Gemeindeversammlungen 19

Gemeinwohl 32, 37, 117, 124, 195, 231

Genf 236, 237

Gesamtänderung der Verfassung 241, 251

Geschworenengerichtsbarkeit 256

Gesetz gegen die Neubildung von Parteien 136

Gesetzesdokumentation 212

Gesetzesinitiative 167, 183, 184, 194, 206, 209, 211, 214, 222, 245, 249, 255

Gesetzesreferendum 205, 209, 210, 212

Gestaltungsbefugnis der Initiatoren 95 Gewaltenteilung 186, 221, 251, 296

Gewerkschaften 176-183, 185, 188, 198

Glarus 210, 238

Gleichheit der Wahl 62

Gleichrang von Sach- und Personalentscheidungen 16

Gleichrang von Volks- und Parlamentsgesetzgebung 116

Gleichschaltung der Länder 135, 136

Glücksspiel 175, 189, 198, 202, 203, 231

Goebbels, Joseph 142

Grundgesetz 11, 13, 14, 16-21, 23-25, $27,32,34,39,42,84,92,115,116$, $122,210,259,274,295,311,315$, 340

Grundprinzipien der österreichischen Bundesverfassung 252 
Grundrechte 59, 84, 108, 186, 247, 251,315

\section{Grundsteuern 171}

Grundverkehrsteuern 175

Habermas, Jürgen 278

Hamburg 28, 40, 50, 56, 58, 61, 90-97, 99, 101-103, 105, 106, 109, 110, $113,117-121,127,129,130,133$, $144,149,151,153,158,161,162$, 197, 299, 307, 310, 311, 315

Hamburger Verfassung 90

Hamburgisches Verfassungsgericht 93, 96, 97, 99, 101, 102, 104, $105,107,108,110,116,119,121$, 125

Hanfinitiative 227, 228

Haushalt 17, 26, 33, 125, 175, 307, 319

Haushaltsvorbehalt 25, 26, 104, 105, 122-124, 303, 309, 317

Haushaltswirksame Volksabstimmungen Siehe finanzwirksame Abstimmungen

Herrschaft auf Zeit 35

Hessen 15, 40-42, 47, 51, 171, 300, 307,314

Heuss, Theodor 23

Himmler, Heinrich 138

Hindenburg, Paul von 22, 140, 142

Hitler, Adolf 14, 21, 132-134, 139, 140, 142-144, 154, 155

Hoffmann-Riem, Wolfgang 92

Hofmann, Hasso 21

Homogenitätsklausel 20, 30, 122

Homosexuelle 75-78, 172, 183, 185, 186, 194, 195, 198

Hooligan-Konkordat 237

Hotel- und Gaststättengewerbe 37

Howell, Carla 175

Hume, David 68
Hundehaltung 235

Hunderennen 194

In Re Marriage Cases 184-186

Indirekter Gegenentwurf 216

Initiative 29-31, 66, 68, 74, 75, 77, 78, 82-84, 93, 94, 97, 99, 102, 104, 106, $118,122,165-172,175-178,182$, $183,186,187,189,191,193-199$, 201-205, 207, 208, 212, 214-217, 219-221, 223-226, 230, 232-235, $238,246,260-262,265,268,278$, 280, 298-301, 305, 306, 314

Initiative and Referendum Institute (IRI) 166

Initiative Industry 170

Instrumentalisierung von Plebisziten 141

Interlaken 240

Interorganrespekt Siehe Organtreue Iowa 184,200

Irland 278

Islam 80

Italien 40, 43, 49, 51, 53, 63, 103, 132 ius cogens 219,220

Jagd von Flugzeugen aus 194

Japan 66

Judikative 17

jugend + musik 232

Jung, Otmar 148, 150, 340

Jura 22, 80, 259

Kalifornien 75-77, 81-83, 88, 103, $112,166-169,171-175,177,179$, 182-185, 187-190, 192-194,

196-198, 200, 202

Kampfflugzeuge 232

Kampfhundeverbote 236

Kant, Immanuel 32 
Kantone 33, 71, 75, 79, 80, 86, 205 , 207-212, 214, 217, 219, 223, 224, 226, 229, 231, 232, 234-240

Kantonsverfassungen 205, 208

Karenzzeit 313

Kasinospielsteuer 190

Keine neuen Tagebaue 162

Kinderkrankenversorgung 187

Kindertagesstätten 32, 239, 304, 309

Komplementärmedizin 230

Konkrete Normenkontrolle 268, Siehe Präventive Normenkontrolle, Verfassungsgerichtsbarkeit

Konkurrenz von Parlaments- und Volksgesetzgebung 249

Konsensdemokratie 23

Konstitutionalisierung 119, 174, 271, 273, 275, 277, 283

Konterlegislatur 97, 99, 102, 103, 111, 115-118, 129

Kopplung von Wahl- und Abstimmungstag 110, 111, 113, 128, 173

Kopplungsverbot 98

Korrektivfunktion 34-36, 194

KPD 47, 136, 150, 151, 153-158

Krankenkassenprämien 230

Kumulieren 113

Landesbetrieb Krankenhäuser 96

Landeshaushalt 104-106, 298, 300, 316 , Siehe Budget, Haushalt

Landesverfassungen 12, 16, 20, 36, 37 , 43, 44, 90, 92, 249-251, 307, 311, 315

Landschaftsinitiative 233

Landsgemeinde 71, 74, 79, 208, 210, 212

Landtag 24, 25, 36, 42, 44, 47, 65, 170 , 248, 249

Landtagsauflösung 16, 45-47

Latinos 76, 172
Lebendiges Wasser 223, 234

Legehennen 194

Legitimationskette 17

Lehrergehälter 193

Lernmittelfreiheit 36

lex-posterior-Grundsatz 116

Lippe 44, 45, 48

Liberale Verfassungstradition 70

Lobbyismus 37

Logit-Modell 147, 154

Lokales Referendum 264

Lübeck 47, 134, 146, 152, 153, 157

Luzern 80, 237, 239

Madison, James 88

Maine 173, 181, 184, 189, 198, 200, 203

Marihuana 188, 194, 195, 203

Massachusetts 75, 83, 166, 175, 184, 188, 194, 195, 200, 203

Massenpetition 299

Maulkorbinitiative 218, 225

Mecklenburg-Vorpommern 299, 300, 302, 307, 311

Mehr Demokratie 49, 50, 57, 58, 94, $95,99,102,103,117,161,162,306$, $311,335,340$

Mehrheit entscheidet 30

Mehrheitsprinzip 29, 40

Mehrwertsteuer 207

Memorialsantrag 212

Menschen- und Bürgerrechte 66, 72

Menschenrechte 66, 67, 69, 72, 81, 84, $86,88,221$, Siehe Grundrechte

Michigan 83, 166, 183, 188, 191, 200, 203

Mietpreiskontrollen 182

Minarettinitiative 220, 234 
Minderheiten 28, 31, 68, 69, 74-77, 79-81, 88, 172, 183, 192, 195, 196, 199

Minderheitenherrschaft 28, 51, 112, 181

Minderheitenschutz 28, 172, 270

Mindestlöhne 27, 329

Mindeststrafen 189

Minoritäten Siehe Minderheiten

Mississippi 167, 200

Missouri 166, 183, 187, 190, 193, 200, 203

Mittelbare Effekte von Volksinitiativen 215

Mitwirkung der Länder an der Gesetzgebung 11

Mobilisierungslast 53

Mobilisierungsrelevante Verfahrensregeln 109

Montagnard-Verfassung 208

Montana 166, 187, 200, 203

München 64, 318

Nachabstimmungsbefragungen 59

Napoleon Bonaparte 269

Napoleon III. 14, 66

National Conference of State Legislatures 166

National Taxpayers Union 175-177

Nationalrat 206, 207, 214, 217, 241, $243,245,246,252-255$, Siehe Bundesversammlung

Nationalversammlung 20, 268

Nationalsozialismus 22, 66, 84, 132, $135,143,145-148,153,155,156$, 158

Nebraska 166, 173, 183, 195, 196, 200, 203

Negatives Stimmgewicht 61, 62, Siehe Erfolgswert

Neuenburg 71
Neukaledonien 264, 268

Neumann, Peter 40

Neumünster 146, 147, 152-155

Neutralitätspflicht 328

Nevada 166, 182, 194, 200, 203

New Hampshire 200

New Jersey 200

New Mexico 201

New York 201

Nidwalden 210, 211, 217

Niederlande 278

Niedersachsen 25, 299, 300, 307, 311

Non-Refoulement-Prinzip 219

Nordrhein-Westfalen 18, 41, 48, 59, 64, 299, 302, 307, 311, 312, 329

Normenkontrolle 32, 85-87, 109

North Carolina 201

North Dakota 173, 174, 176, 177, 188, 195, 201, 203

Novemberrevolution 70

NS-Plebiszite 133

NSDAP 22, 133-137, 141-146, $153-155,158$

Obligatorisches Referendum 15, 206

Obwalden 217

Offenlegungsvorschriften 197

Öffentliche Wahlkampfunterstützung 181

Öffentlichkeitsarbeit des Staates im Vorfeld eines Volksentscheids 333

Ohio 166, 189, 198, 201, 203

Oklahoma 183, 201

Oldenburg 47, 134

Opposition 24, 29, 34, 36, 46, 65, 85, $118,129,136,139,141,144,150$, $151,153,154,156,194,195,261$, 298, 306 
Oregon 83, 166, 173, 174, 176, 178, 181, 189, 192, 193, 198, 200, 201, 203

\section{Organkompetenz 104}

Organstellung des Volksgesetzgebers $26,108,323$

Organtreue 97, 98, 102, 116, 319, 320, 323

Österr. Verfassung aus 1920253

Österr. Verfassungsgerichtshof 244, 247-251

Österreich 103, 136, 140, 238, 241, 247, 248, 252-255, 257, 258, 277

ÖVP 256

Panaschieren 113

Parkraumbewirtschaftung 163

Parlament 15, 18, 24, 27, 46, 47, 51, 67, $70,71,75,78,80,82-86,89,98,101$, 111-116, 123, 124, 167, 169-171, 174, 179, 181, 184, 187, 189, 192-194, 206, 212, 213, 215, 219 , $220,222,226,229,230,236,237$, $245,251,253,254,257,260,262$, 263, 268, 269, 278-281, 302-304, 314, 321, Siehe Abgeordnetenhaus, Bürgerschaft, Bundestag, Bundesversammlung, Landtag, Nationalrat, Nationalversammlung

Parlamentarischer Rat 23

Parlamentsauflösung 43

Parlamentsidealisierung 37

Partei Rechtsstaatliche Offensive 96

Parteien 35, 36, 38, 47, 52, 54, 59, 65, $113,114,129,136,138,139,147$, 180-182, 190, 207, 215, 224, 227, $246,248,254,256,257,275,276$, 279,281

Parteistrategen 52, 54, 55

Partialrevision 206, 209

Partizipation 51, 54, 94, 181, 194, 257, 280, 284
Paulskirche 20

Payday Loans 179

Peine, Franz-Josef 118

Pennsylvania 201

Periodic Convention Question 169

Pflege 187

Plebiszit 13, 14, 19, 23, 38, 46, 104, $116,124,125,127,128,137,140$, $141,148,150,152,153$

Polarisierung 23

Police Power 165

Politikverdrossenheit 34

Popular Referendum 168

Prämie für jeden Demagogen 23

Präponderanz der repräsentativen Demokratie 17, 18, 24, 123, 241

Pratteln 69

Präventive Normenkontrolle 109, 173, Siehe Normenkontrolle, Verfassungsgerichtsbarkeit, Vorprüfung

Preußen 46, 47, 70, 135

Primärrecht 278, 279, 283

Primat der direkten Demokratie 218

Privacy 186

Private Krankenversicherungen 187

Pro Reli 50, 58, 162, 312, 333

Propaganda 132, 135, 141, 142, 157, 225

Proposition 6377

Proposition 8 174, 183-186, 194, 196, 198, 203

Quorum 15, 28-30, 36, 40, 46, 48, 49, 51-54, 58, 65, 72, 94, 95, 102, 109, 111-114, 117, 118, 120, 126-129, 161-163, 168, 196, 211, 262, 268, $270,283,284,301,302,304-306$, $312,314,316,320$

Randständige 237

Rauchverbot 163, 231, 237 
Recht auf Heirat Siehe Eheschließung Rechtsprechung der Landesverfassungsgerichte 11, 26, 37, 105, 121

Rechtsstaat 66, 68-71, 81, 87, 242, 243, 251

Referendum 14, 15, 26, 67, 68, 72, 74, $80,82,83,87,93,112,117,118,120$, 130, 169, 191, 192, 201, 204-207, $210,213,214,222,223,228,229$, 235, 236, 247, 259-270, 279, 313, 314

Referendum auf Minderheitsinitiative $262,264,267,268$

Refolution 90, 103, 115, 130

Reichspräsident 22, 142

Reichstag 22

Relationales Zustimmungsquorum 112

Repräsentanten 13, 27, 29, 32-34, 37-39, 222, 242

Repräsentativ-demokratisches Baugesetz 249

Repräsentative Demokratie 12, 38

Repräsentative Elemente der direkten Demokratie 38

Republik 13, 22, 32, 139, 241, 251, 254, 257, 259, 260, 263

Republikanische Partei 180-182

Responsivität 35, 103, 195

Rheinland-Pfalz 41, 43, 48, 57, 60, 64, 298-300, 302, 307

Rhinow, R.A. 74

Rhode Island 201

Röhm, Ernst 140

Rot-Grün 42

Rousseau, Jean-Jacques 38, 115, 208

Rücknahme einer Vorlage 107

Rückzugsklausel 215

Rudi-Dutschke-Straße 57

Rumänien 228
Rundfunk-Reform 254

Rüstungsausgaben 208

référendum législatif 259, 262, 264

Saarland 31, 54, 300, 307, 311, 312, 315,323

Sachsen 40, 45, 48, 50, 57, 116, 155, 300, 307, 310-312

Sachsen-Anhalt 109, 299, 300, 307, 311

Sachunmittelbare Demokratie 14

Sachverstand der Bürger 27

Salzburg 250

Sammlungsfrist 211, 215, 217, 231, 232

Schaumburg-Lippe 45, 47

Schein von Normalität 137

Schleswig-Holstein 50, 57, 121, 132-135, 138-140, 143, 146, 150, 151, 153-158, 161, 299-302, 307, 311

Schubert-Praxis 221, 222

Schulwesen 235

Schwächung der parlamentarischen Demokratie 24

Schwangerschaftsabbruch $75,78,190$, 191

Schwarzburg-Sondershausen 47

Schwarzenegger, Arnold 179, 180, 191

Schweiz 14, 23, 31, 33, 40, 63, 65-75, $79,80,82,84-87,89,103,118,124$, $167,205,210,218-220,224,225$, 227-231, 234-236, 238, 239

Schweizerische Bundesverfassung 129

Schwerfälligkeit der Volksgesetzgebung 27, 116, 123

Sekundärrecht 278, 279, 283

Senat 90-92, 96-101, 103, 107, 108, $111,119,121,130,179,265,304$, 
308-312, 315, 319-324, 328, 332, 333

Senkung des Stimmrechtsalters 238

Sicheres Wohnen im Alter 232

Sizemore, Bill 176, 178, 181, 192, 198

South Carolina 201

South Dakota 166, 167, 179, 180, 190, 191, 195, 201, 204

SPD 35, 42, 47, 61, 92, 94, 95, 151, 153-158, 340

Sperrwirkung 97, 110, 116, 118, 171, 268, 313, Siehe Konterlegislatur SPÖ 247, 256

St. Gallen 80, 209, 237, 238

Staatslotterie 189

Staatspräsident 259-263, 266, 267

Staatsqualität der EU 271

Staatsrechtslehre 21, 22, 25, 105, 242

Staatsvertragsreferendum 206, 215

Ständemehr 79, 217, 223, 229, 231

Standesinitiative 212

STATT-Partei 92

Status von Quasi-Volksgesetzen 120

Statutory Initiative 167

Sterbehilfe 175, 190, 191, 195, 204

Stern, Klaus 12

Steuererhöhungsinitiativen 177

Steuergerechtigkeits-Initiative 233

Steuersenkungsinitiativen 175

Steuerwettbewerb 236

Stimmensplitting 51

Stopp-Offroader-Initiative 233

Strauß, Franz-Josef 33

Strauß-Fernsehen 24

Studiengebühren 32

Supreme Court 82, 85, 168, 182-186, 190-192, 196

SVP 67, 224
Synchronisierung des Legitimationsniveaus 113

Tabakinitiative 232

Teilrevision der Verfassung 71, 212, 214, Siehe Partialrevision, Verfassungsänderungen

Tempelhof 48, 50, 58, 164, 302, 319, 320, 324-326

Tennessee 201

Territorialplebiszit 14, 19

Territorien in Übersee 259, 264

Texas 201

Theorie des Wählerverhaltens 73

Thüringen 16, 28, 33, 62, 94, 103, 161, 299, 300, 307, 311, 347

Thüringer Verfassungsgerichtshof 16 , $19,23-25,27,28,30,33,37,124$

Todesstrafe $66,69,70,75,78,81-83$, 88

Totalrevision 71, 205, 207, 208, 218

Transrapid 317

Treibhausgasemissionen 233

Türkei 262

Überarbeitung der Vorlage 107, 124, 126

Überforderung der Bürger 26

Umweltschutz 75, 175, 193, 198, 240

Unentbehrlichkeit des Wahlakts 30

Unmittelbarkeit der Wahl 62

Unterschriftensammlung 102, 110, 211

Unverjährbarkeitsinitiative 222, 226, 227

\section{Uri 238}

Urnenabstimmungen über Einbürgerungen 67

USA $14,23,31,33,40,63,65,66,69$, 73, 75, 78, 79, 82, 83, 87, 124, 165, 
$167,168,170,173,174,185,186$, 190, 198, 199

USPD 47

Utah 201

Verbände 52, 145, 257, 280, 283

Verbandskompetenz 104

Verbindlichkeit von Volksentscheiden 102, 119, 130, 263

Verbot von Kriegsmaterial-Exporten 234

Verbot von Leerverkäufen 179

Verbrechensopferrechte 188, 198

Verfassunggebung 15, 20, 278

Verfassungsändernde Volksgesetzgebung 41

Verfassungsänderungen $15,43,56,58$, $72,90,91,93-95,99,102,103$, $112-114,120,121,127,167,168$, $173,174,182,184,186,196,200$, $221,252,256,265,299,304,313$, 315

Verfassungsdurchbrechung 56

Verfassungsgericht des Landes Brandenburg 318

Verfassungsgerichtliche Kontrolle 32, $168,174,265,266$, Siehe Normenkontrolle

Verfassungsgerichtsbarkeit $11,16,40$, 69-71, 84-89, 105, 121, 123, 124, $126,127,210,218,268$

Verfassungsinitiative 82, 86, 166, 167, $171,172,174,179,182,183,186$, 190, 196, 202, 205, 209, 211, 308, 313,315

Verfassungsorgantreue $310,313,328$, Siehe Interorganrespekt, Organtreue

Verfassungsreferendum 28, 51, 167, 171-174, 205, 207, 209, 300, 304, 313

Verkaufsteuer 187

Verkehrsprojekte 177
Vermont 201

Verschuldung 33, 169

Vertrag von Lissabon 37, 72, 252, 276 , 278-280, 283, 284

Verwahrungsinitiative 66, 67, 217-221, 227

Verwaltungsreferendum 210

Virginia 201

Völkerrechtswidrige Volksinitiativen 219

Volksabstimmung 15, 19, 24, 32, 42, $65,76,80,112,114,132,133$, 136-141, 144-147, 150, 154, 207, 210, 217-220, 225, 237, 239-241, $243,246-253,257,260,262,263$, $265,266,269,270,297,300,314$. 321

Volksanregung 206, 213

Volksauftrag 212

Volksbefragung 16, 209, 242, 243, 245, 246, 264

Volksbegehren 15, 20, 25-28, 30, 32, $35,36,41,42,56,59,65,68,84$, 90-104, 106, 107, 110, 111, 120-123, 127, 137, 161-163, 185, $207,218,221,243,245,248-250$, 253-255, 258, 297-302, 305-312, $315,317,318,321,323,324,329$, 333,335

Volksdiskussion 210

Volksentscheid 15, 16, 22, 25-27, 30, $31,35,36,40-44,47,50-53,55-60$, $63,68,70,80,84,90-102,105-108$, $110-113,116-118,120-122$, $126-130,137,161,163,168,171$, $174,176,194,196,214,222,259$, 261, 263, 267, 296-300, 303, 305, $306,308,309,311-313,319-321$, $323,324,328,333,335$

Volksgemeinschaft 131, 133, 136, 138, $144,148,157$ 
Volksinitiative 15, 59, 67, 71, 92-97, 99, 100, 102, 104-108, 113, 117, $118,120,127,129,167,168,171$, 197, 205-207, 210-217, 219, 221-223, 227, 229-234, 236-238, 261, 297-300, 305, 324, 335

Volksmehr 79, 217, 223, 229, 231

Volksmotion 212

Volkspetition 94, 106, 299

Volksrechte 19, 66, 68, 71, 72, 75, 77, 79, 81-84, 87-89, 205-207, 218, 231

Volkssouveränität 13, 16, 30, 38, 87, 208, 209, 225, 339

Volksvorschlag 210, 211

Volkswahl von Amtsträgern 14

Voluntary Referendum 169

VolXUni 99, 104, 105

Völkerrecht 14, 67, 68, 86, 87, 89, 216, 219-222, 225, 227, 274

Vorabkontrolle 266, 267, 269

Vorarlberg 248

Vorprüfung 211, 213, 215, 231

Vorruhestandsleistung 230

Vorwahlen 173, 181, 195, 204

Vorwirkung von Volksbegehren 36

VOX-Analyse 214

Waadt 209, 210, 239

Wahlanalyse 156, 158

Wahlbeteiligung 29, 34, 113, 114, 128, $137,145,147,150,152,153$

Wahlen 11, 13, 16-18, 23, 25, 28, 29, $47,55,73,83,97,98,102,111-113$, $119,122,126,128,131-137,141$, $147,150,153,157,158,173,174$, $180,181,194,215,241-243,255$, $275,283,295,312,314,318,321$, 324-328

Wählermilieu 156
Wahlfälschung 63, 145, 146, 150

Wahlgeheimnis 157

Wahlkampf 27, 29, 68, 111, 141-143

Wahlkampfspenden 180, 198, 202, 204

Wahlkreisneueinteilung 180, 195, 198

Wahlrechtsgrundsätze 59

Wahlschleppdienste 139, 152, 157

Washington 83, 166, 170, 174, 177, 183, 187, 191, 201, 204

Washington D.C. 83, 170

Weber, Max 23

Wegweisungsartikel 237

Weiler, Joseph 283

Weimarer Reichsverfassung 20, 22, 26, 42, 44, 56, 105, 117, 129

Weimarer Republik 43, 44, 47, 48, 54, $56,65,84,134,135,137-139,142$

West Virginia 201

Western Phenomenon 174

Wiederholungsverbot 306

Windenergieanlagen 239

Wisconsin 201

Wyoming 201

Young-Plan 22, 44

Zerfaserung der Verfassung 222

Zürich 79, 211, 212, 223, 235, 236, 238, 239

Zustimmungsmehrheit 113, 120

Zustimmungsquorum 30, 40, 41, 43, $45,46,50-52,55-58,64,65,95,99$, $112,114,115,120,128,198,202$

Zweistufiges Modell der Volksgesetzgebung 94

Zweitwohnungen 234

Zwischenkriegszeit Siehe Weimarer Republik 